\section{Fluorescence in situ hybridization analysis with LIS1 specific probes reveals a high deletion mutation rate in isolated lissencephaly sequence}

Daniela T. Pilz, MD, Michelle E. Macha, BS, Kathrin S. Precht, BA, Ann C. M. Smith, MA, William B. Dobyns, MD, and David H. Ledbetter, PhD

Purpose: Recent revision of the lissencephaly critical region on chromosome 17p13.3 and confirmation of LIS1 as the causative gene for classical lissencephaly has allowed the development and application of fluorescence in situ hybridization (FISH) probes corresponding directly to this gene. Method: We have analyzed patients with isolated lissencephaly sequence (ILS) by FISH with probes at D17S379, an anonymous locus distal to LIS1, and with LIS1 specific probes. Results: In 110 patients with ILS, a deletion at D17S379 was detected in $23.6 \%$. Of those patients without a deletion, 32 were available for further study with LIS1 probes. Deletions were found in eight additional individuals. Conclusion: The overall deletion mutation rate detectable by FISH with LIS1 probes is $\approx 40 \%$. This rate is significantly higher than the deletion rate observed at D17S379. This indicates that FISH studies using probes specific to LIS1 should be undertaken as the initial diagnostic assay for the evaluation of patients with ILS, and the high frequency of deletions raises the possibility of "hotspots" for chromosome breakage in this region.

Keywords: Lissencephaly, LIS1 gene, FISH

\section{INTRODUCTON}

Classical lissencephaly is a disorder of neuronal migration characterized by agyria or pachygyria with an abnormally thick cerebral cortex. It merges with subcortical band heterotopia (SBH), also known as "double cortex", to form the agyria-pachygyria band spectrum of cortical malformations. Three major syndromes have been identified with classical lissencephaly or SBH: Miller-Dieker syndrome localized to chromosome $17 \mathrm{p} 13.3$; isolated lissencephaly sequence (ILS17), associated with abnormalities of 17p13.3; and X-linked lissencephaly (ILSX), in which affected males have the agyric/pachygyric form of classical lissencephaly and carrier females show SBH.,

Patients with ILS have classical lissencephaly of variable severity with few dysmorphic features. These include a square forehead, short nose, thin upper lip, and small jaw. They are profoundly retarded, and the vast majority have seizures. ${ }^{3,4}$ Visible deletions of $17 \mathrm{p} 13.3$, seen in more than $50 \%$ of patients with Miller-Dieker syndrome, have never been reported in patients with ILS ${ }^{5}$ and were not seen in our present series; however, since submicroscopic deletions of this region were first reported in $1991,{ }^{6,7}$ they have been identified in an increasing number of patients. Two series using cosmid probes corresponding to D17S379 for fluorescence in situ hybridization (FISH) studies showed a detection rate of $13 \%{ }^{8}$ and $18 \%$, respectively, for ILS.

In 1993 a gene, LIS1, was mapped to $17 \mathrm{p} 13.3$ and proposed as a candidate gene for lissencephaly. ${ }^{10}$ Subsequently, it was found that one of the CDNA clones used in the determination of the $5^{\prime}$-end of LISI was chimeric and, in fact, was derived from a more telomeric gene on $17 \mathrm{p} .{ }^{11}$ The correct $5^{\prime}$-end of LIS 1 has now been identified and the critical region for lissencephaly revised. ${ }^{12}$ Mutations within this gene were identified in several nondeletion patients with ILS, thus providing the final confirmation that $L I S 1$ is the $17 \mathrm{p}$ lissencephaly gene. ${ }^{13}$ LIS1 is known to encode a subunit of the brain platelet-activating factor acetylhydrolase (PAFAH), ${ }^{14}$ although its precise role in neuronal migration has yet to be determined.

In view of the revised map of the lissencephaly region, ${ }^{12}$ we have developed further genomic probes partially or completely spanning the LIS1 gene for deletion assessment by FISH. We present our FISH data on 110 patients with ILS and demonstrate that the LIS1 probes significantly increase the deletion detection rate in these patients to $\approx 40 \%$.

\section{MATERIALS AND METHODS}

\section{Subjects}

All 110 patients, 60 males and 50 females, were evaluated by one of the authors (D.T.P. or W.B.D.) to confirm the diagnosis of ILS. This included a review of the clinical data and the cranial CT or MRI scan in all cases. If the patient could not be examined in person, photographs were used to document the facial phenotype. Details of the phenotype, including appearance, radiological and pathological abnormalities, and clinical course observed in ILS, have been published elsewhere. ${ }^{1,3,4}$ Blood samples from the probands, and in some cases their parents, were obtained with informed consent. All protocols were approved by the appropriate Human Subjects Committee Institutional Review Board.
From the Department of Human Genetics, The University of Chicago, Chicago, Illinois; the National Human Genome Research Institute, National Institute of Health, Bethesda, Maryland; and the Department of Neurology, University of Minnesota Medical School, Minneapolis, Minnesota.

Address correspondence to: David H. Ledbetter, PhD, Department of Human Genetics, The University of Chicago, 924 East 57th Street, Chicago, II 60637. E-mail: dhl@genetics. uchicago.edu

C) 1998 Genetics in Medicine. All rights reserved. 1098-3600\$0.00 


\section{Probes}

The probes utilized and discussed in this paper are shown in Figure 1. Patients initially were screened for deletions at D17S379 with a small cosmid contig (c197-9, c197-4, and c197-2) used as a pooled probe of $\approx 80 \mathrm{~kb}$ as described previously. ${ }^{8}$ Additional genomic clones proximal to LIS1 included c136F10/93E5 and c115B3. These were used before the identification of the LIS1 gene and corresponding genomic clones. Of several LIS1-specific clones, c120A7 and PAC-95H6, estimated to be $\approx 35 \mathrm{~kb}$ and $\approx 110 \mathrm{~kb}$ in size, respectively, were primarily used. The PAC clone $95 \mathrm{H} 6$ contains both the $5^{\prime}$ - and $3^{\prime}$ ends of the LISI gene. Details and availability of the clones have been published elsewhere. ${ }^{12}$

\section{Fuorescence In situ hybridization}

Chromosome preparations were made from peripheral blood or lymphoblastoid cell lines by standard methods. FISH analysis was carried out as described previously ${ }^{12}$ with minor modifications.

Cosmid and PAC DNA, labeled with digoxigenin11-deoxyuridine triphosphate (dUTP; Boehringer Mannheim, Indianapolis, IN) were precipitated in ethanol with $30 \times$ excess of human Cot-1 DNA and herring testis DNA (Gibco-BRL) and resuspended to a final concentration of $20 \mathrm{ng} / \mu \mathrm{L}$ in hybridization solution $(50 \%$ formamide $/ 2 \times$ saline sodium citrate (SSC) $/ 10 \%$ dextran sulfate). After addition of chromosome $17 \alpha$-satellite probe labeled with biotin-16dUTP (Boehringer Mannheim), the probes were denatured at $76^{\circ} \mathrm{C}$ for 7 minutes and preannealed at $37^{\circ} \mathrm{C}$ for 15 minutes.

The probe mixture $(6 \mu \mathrm{L})$ was applied under a 22 $\mathrm{mm} \times 11 \mathrm{~mm}$ coverslip, and slides were incubated in a moist chamber at $37^{\circ} \mathrm{C}$ for 16 hours. The probes were detected in $60 \mu \mathrm{L}$ of rhodamine anti-digoxigenin (Boehringer Mannheim) at $1 \mu \mathrm{g} / \mu \mathrm{L}$ and fluorescein isothiocyanate (FITC)-avidin DCS (Vector Laboratories, Burlington, CA) at $5 \mu \mathrm{g} / \mu \mathrm{L}$. At least 10 metaphases were analyzed for each hybridization using a Zeiss Axiophot microscope with filters to detect 4,6diamidino-2-phenylindole (DAPI), fluorescein isothiocyanate, and rhodamine separately as well as a triple band pass filter (no. 83000, Chroma Technology Corp., Brattleboro, VT) to detect signals simultaneously. Images were collected and merged using a cooled CCD camera (KAF 1400 Photometrics, Tuscon, AZ) and
IP Lab Spectrum (Signal Analytics Corp.) or Smart Capture software (Vysis, Inc., Downers Grove, IL).

\section{RESULTS}

Of the 110 patients tested with probes at D17S379. deletions were detected in 26 patients (23.6\%). This deletion frequency is higher than in the previous twe series on smaller numbers of patients ${ }^{8,9}$ due to thy use of the more stringent diagnostic criteria of ILA the last few years. Further analysis in 16 of these deleted patients with probes located centromeric to the LIS1, or more recently with the LIS1 gene probe. c120A7, PAC-95H6, and c37E9 (Fig. 1), showed evis dence of a deletion of the entire LIS1 in 15 individuals (Fig. 2, A and B), whereas one represented $\mathrm{a}$ partial deletion of the gene (discussed below).

Of the patients not deleted at D17S379, 32 were available for study with the LIS1 probes C120A7, PAC 95H6, and c37E9. Deletions were found in an additional eight of these patients (Fig. 2, C and D). Sever of the children had a deletion of the entire gene whereas one had a partial LIS1 deletion (see below)

Overall, deletion status for PAC-95H6, spannin $L I S I$, was tested directly or could be inferred fron FISH data on flanking probes in a total of 48 patients of whom 16 were deleted and 32 were not deleted at D17S379.

A complete deletion of $L I S 1$ was identified in 22 of 48 cases. A partial gene deletion was observed in two patients. They had previously shown to be deleted with probes telomeric or centromeric to the LISH gene (unpublished data). The first child was deleted at D17S379 (Fig. 3A). Studies with PAC-95H6 gavi a much weaker, rather than completely absent, sig nal on one 17 homologue, indicating a partial dele: tion of the gene. Consistent with this, FISH showe no deletion with c120A7, which corresponds to the 3 -end of the gene. The second child was not deleter at D17S379 (Fig. 3B); however, he was found to $t$ mosaic for a deletion ( $80 \%$ of cells) with probe. c93E5/c136F10 and 115B3 centromeric to the $L I 5$ : gene and the LIS1 probe c120A7. The PAC-95H6 sif nal was visible on both 17 homologues, but one sit nal was significantly weaker than the other in $\approx 80$ of cells (Fig. 3B). Phenotypically, he had generaliz: pachygyria indistinguishable from patients witti nonmosaic LIS1 deletion. In both patients heteromorphism in the size of the $\alpha$-satellite sig

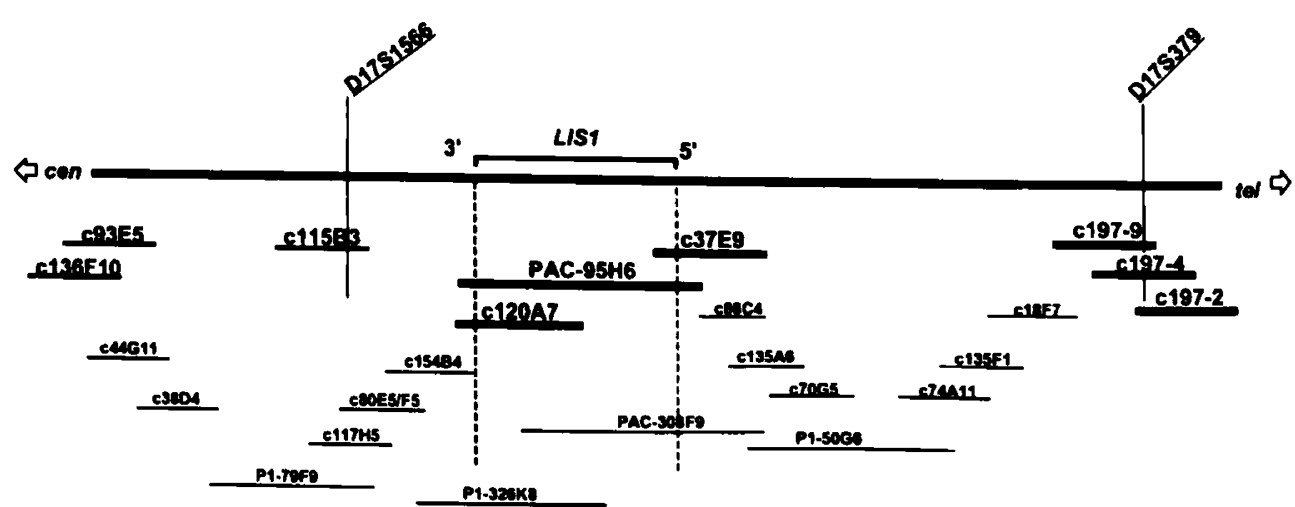

FE 1. Map of the lissencephaly critical region in $17 \mathrm{p} 13.3$ adapted from Chong et al. ${ }^{12}$ (not drawn to scale). A complete cosmid, $\mathrm{P} 1$ and PAC contig was constructed from D17S379 $(\approx 150-200 \mathrm{~kb}$ telomeric to LIS1) to cosmid c136F10 $(\approx 150 \mathrm{~kb}-200 \mathrm{~kb}$ centromeric to LISI) The orientation and position of the LIS1 gene is shown within the contig. Cosmid and PAC clones used in FISH studies are bolded, including cosmids c197-9, c197-4, and c197-2 located at D17S379, and LIS1 probes PAC-95H6, c120A7, and c37E9. 

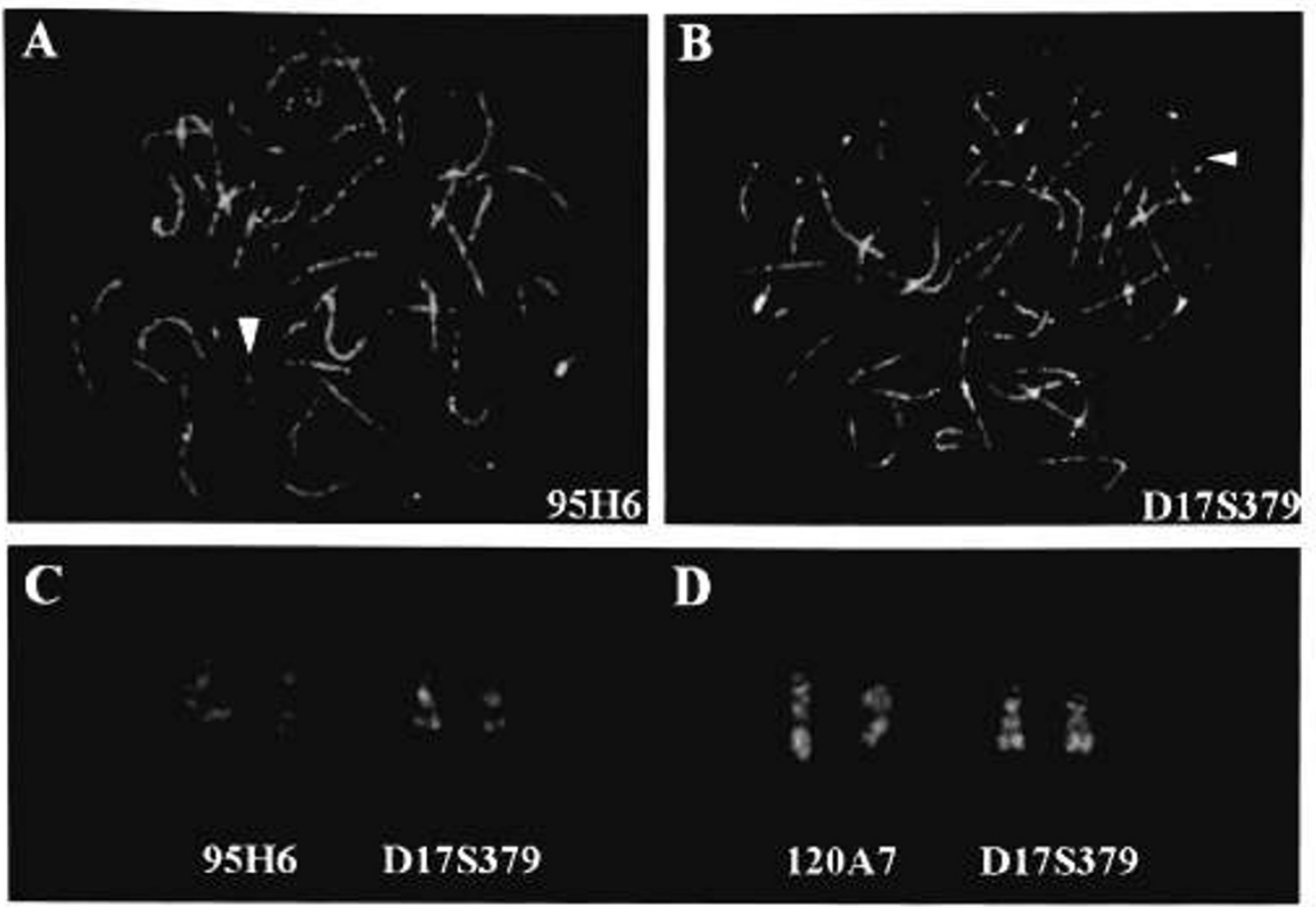

Fig. 2. FISH analysis comparing D $17 S 379$ cosmids to LIS1 specific clones. Panels A and B show FISH studies on the same patient, who demonstrated a deletion on one homologue (white arrow) with both PAC95H6 (A) and at S379 (B). Panels C and D show two other ILS cases who were not deleted at S379, but did show a deletion with LIS1 probes PAC95H6 (C) and c120A7 (D).
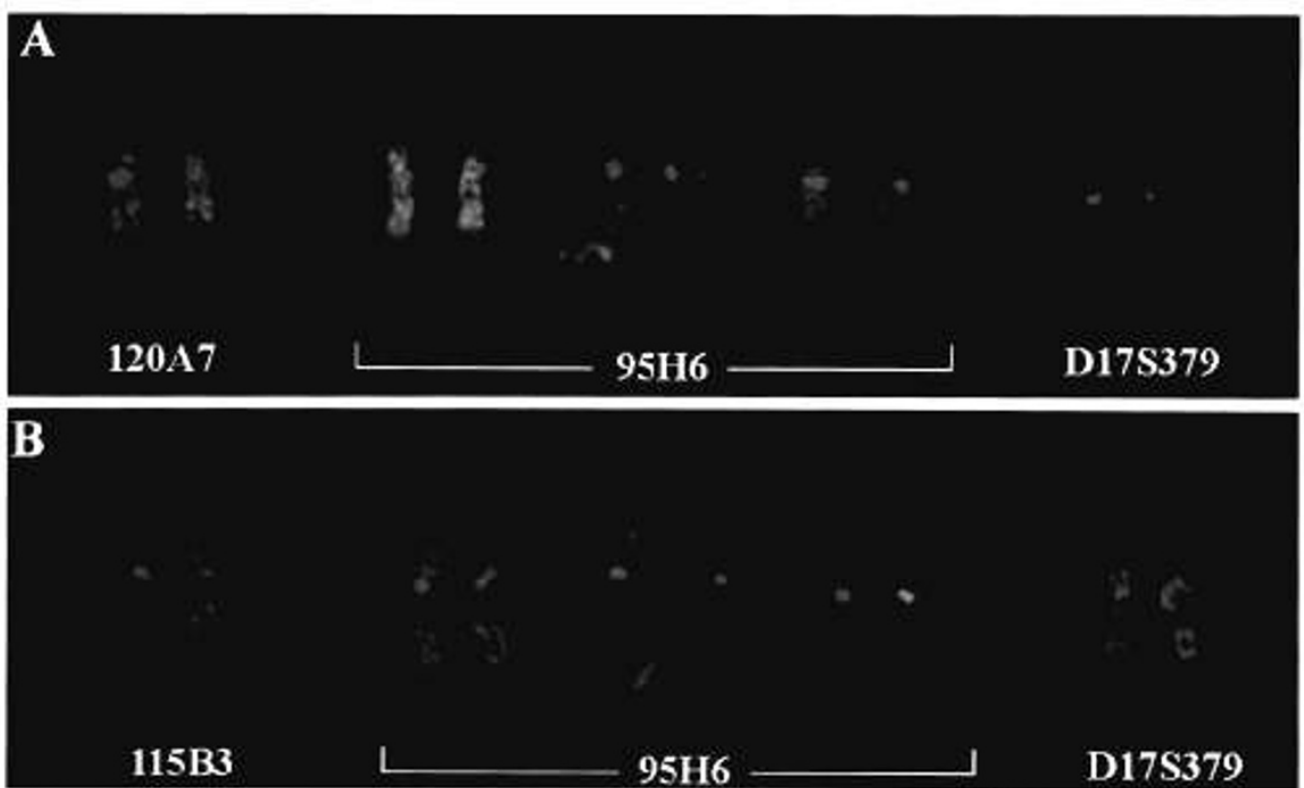

FI. 3. FISH analysis of two patients demonstrating partial deletions of the LIS1 gene. The first patient (A) was deleted at S379, and with PAC95H6, a weaker signal was found on one 17 homologue (on the right in each pair). A partial deletion of LISI was confirmed by finding normal signals on both homologues with c120A7 at the $3^{\prime}$-end of the gene. The second patient (B) had a mosaic deletion in $80 \%$ of cells with c115B3, had a weaker signal with PAC $95 \mathrm{H} 6$ on one 17 homologue (on the right in each pair) in $80 \%$ of cells, and was not deleted at S379. These findings are also compatible with partial deletion. In both cases, a heteromorphism was present in the size of the $17 \alpha-$ satellite signal (green), more prominent in the second case (B). For both, the deleted 17 homologue or the homologue with the weaker FISH signal corresponded consistently to the homologue with the smaller $\alpha$-satellite signal.

on the two 17 homologues assisted in interpretation (more evident in case 2, Fig. 3B). In each case, the homologue with the smaller $\alpha$-satellite signal was the deleted chromosome 17 and the one with a weaker signal when analyzed with PAC-95H6. Although we would not base a final diagnosis solely on the data from PAC-95H6, a significantly weaker signal seen on one chromosome 17 should alert an investigator to the possibility of a partial gene deletion and warrant further FISH or molecular testing.

Overall, deletions at D17S379 were observed in 26 (23.6\%) of 110 patients with ILS. The LISI-specific probes detected eight additional deletions in 32 nondeleted patients $(25 \%)$. We estimate that among the 84 patients not deleted at D17S379, deletions would be detected in approximately 21 if all could be tested 
with LIS1 probes. This would correspond to a deletion detection rate of $43 \%$, considerably higher than that for D17S379.

Of the 34 of 110 patients in our study who were found to be deleted, a slightly higher percentage of deletions was detected in females $(17$ of $50 ; 34 \%)$ compared with males ( 17 of $60 ; 28.3 \%$ ), but was not statistically significant $(0.50<P<0.75)$.

\section{DISCUSSION}

Final confirmation of $L I S 1$ as the causative gene in classical lissencephaly and revision of the critical region in 17p13.3 has allowed the development of a series of genomic probes corresponding directly to the gene. Using these clones as FISH probes greatly increased the detection rate of deletions in patients with ILS. PAC-95H6, which completely spans LIS1 and contains both the $5^{\prime}$ - and $3^{\prime}$-ends of the gene (Fig. 1), is the most informative single probe for the identification of deletions in ILS patients. Some partial deletions of the LISI gene may not be detected with a single FISH probe. In our series, two patients showed significantly weaker, rather than absent, hybridization to one 17 homologue with PAC-95H6. An alternative strategy for deletion detection would be to use two separate clones representing the $5^{\prime}$ - and $3^{\prime}$-ends of the LIS1 gene, e.g., c37E9 and c120A7, which would permit detection of some cases of a partial deletion. Since the present data indicate that most patients are deleted for the entire LIS1 gene, it is probably most cost-effective for the initial clinical laboratory evaluation to be performed by analysis of a single probe.

Among the patients with ILS in whom we did not detect a deletion with FISH, smaller deletions or point mutations of the LISI gene cannot be excluded. Intragenic mutations have so far been reported in eight patients. ${ }^{13,15}$ The majority of these mutations (four frameshift, one nonsense, two splice site, and one missense) result in a truncated protein. This observation, coupled with the comparatively high deletion rate in ILS in our study, indicates that there is likely to be an ascertainment bias toward more severely affected patients. Patients with less severe cerebral malformations, such as generalized or partial pachygyria, may not be commonly recognized as part of the classical lissencephaly spectrum. If so, the diagnosis of ILS may be missed, and the patients may not be referred for evaluation.

Another cause of classical lissencephaly is mutations of XLIS (aka DCX). ${ }^{16,17}$ A girl with classical lissencephaly and a de novo X;2 translocation, ${ }^{3}$ and several families in which affected males had classical lissencephaly (whereas first-degree female relatives had $\mathrm{SBH})^{18,19}$ suggested an X-linked form of classical lissencephaly (XLIS/SBH). Mapping of the translocation breakpoint in the above patient to $\mathrm{Xq} 22.3$ and linkage of families with XIIS/SBHDC to an interval on the $\mathrm{X}$ chromosome containing the breakpoint ${ }^{20,21}$ led to the identification of the abovementioned XLIS gene. Mutations were reported in seven familial cases of ILSX/SBH and in five sporadic females with $S B H .{ }^{16,17}$ This implied that some of the males with ILS, in whom no deletion of LIS1 is detected, may have mutations of the XLIS gene. We recently confirmed this in a study of XLIS in males with sporadic ILS. ${ }^{22}$ In terms of counseling, the possibility of X-linked lissencephaly will always have to be considered in a nondeleted male with the diagnosis of ILS, particularly if there is a history of seizures or learning difficulties in his mother or other first degree female relatives, because these are features associated with subcortical bands in carrier females. Our current studies comparing the lissencephaly in boys with ILSX to children with ILS17 and LIS1 mutations, show consistent differences on neuroimaging, which help suggest which gene is involved. ${ }^{22}$ So far all changes involving XLIS have been intragenic mutations. ${ }^{16,17}$

Our observations of several children with complex lissencephaly phenotypes indicate, that other autosomal forms of classical lissencephaly exist, although they appear to be less common than those associated with alterations of LIS1. Examples include the children described by Ramer et al. $^{23}$ with classical lissencephaly and several additional malformations and those with classical lissencephaly and significant congenital microcephaly. ${ }^{1}$

Deletions in ILS have so far always been de novo; although, when found, parental studies are recommended to exclude the unlikely event of a balanced insertional translocation. Phenotypically, there was no apparent difference between the patients who were also deleted at D17S379 and those deleted only for $L I S 1$. The recurrence risk associated with de novo deletions is very low, mainly conferred by the possibility of germline mosaicism which has not yet been reported in association with this condition. Ultrasonography is not a reliable method to detect classical lissencephaly during pregnancy, particularly in the first and second trimesters. ${ }^{24,25}$ Therefore, detecting such a deletion in an affected child, not only confirms the diagnosis but the very low recurrence risk is reassuring to a couple wishing to extend their family. If requested, accurate prenatal diagnosis by FISH analysis can be offered.

The recurrence risk for parents of children with ILS and no known deletion or mutation of LISI appears to be low. Our previous study of 60 patients with 41 siblings cited a recurrence risk of $7 \% .{ }^{3}$ This figure was based on the observation of three affected siblings from two families out of the total of 41 siblings. Reviewing the data from that paper we determined that the proband and the affected sibling from one of the families was classified incorrectly as having ILS. The other family consisted of three affected brothers. We now suspect that these three boys had $\mathrm{X}$-linked lissencephaly, but have not yet been able to confirm this. No affected siblings (exact number not determined) were identified in any of the families of the 110 patients reported here. Therefore, we estimate that the recurrence risk for ILS, based on this series and our experience, should be significantly less than $5 \%$, especially for families with an affected daughter. The risk for families with affected boys is likely to be slightly higher, because X-linked lissencephaly is a possibility.

The development of LIS 1 gene-specific probes for FISH deletion studies in patients with ILS has increased the detection rate allowing accurate counseling in a greater number of patients. The single clone PAC-95H6 contains the entire LISI gene and will detect all cases of complete gene deletion, making it the most efficient single probe for clinical investigation of classical lissencephaly patients.

\section{Acknowledgments}

We would like to thank all of the families who participated in this study, as well as the Lissencephaly 
Network (U.S.) and the Lissencephaly Contact Group (U.K.) for their help and support. We would also like to thank our colleagues in the Clinical Cytogenetics Laboratory at the University of Chicago for their expert technical support.

\section{References}

1. Dobyns WB, Truwit CL. Lissencephaly and other malformations of cortical development: 1995 update. Neuropediatrics 1995;26:132-147.

2. Dobyns WB. Chromosome $X$ and 17-linked lissencephaly (smooth brain) syndromes. Ment Retard Dev Dis Res Rev 1996;2:118-121.

3. Dobyns WB, Elias ER, Newlin AC, Pagon RA, Ledbetter DH. Causal heterogeneity in isolated lissencephaly. Neurology 1992;42:1375-1388.

4. Pilz DT, Quarrell OWJ. Syndromes with lissencephaly. J Med Genet 1996;33:319-323.

5. Dobyns WB, Reiner O, Carrozzo R, Ledbetter DH. Lissencephaly: a human brain malformation associated with deletion of the LIS1 gene located at chromosome 17p13. JAMA 1993;270:2838-2842.

6. De Rijk-van Andel JF, Catsman-Berrevoets CE, Halley DJ, Wesby-van Swaay E. Niermeijer MF, Oostra BA. Isolated lissencephaly sequence associated with a microdeletion at chromosome 17p13. Hum Genet 1991;87:509-510.

7. Ledbetter DH. Microdeletions of chromosome $17 \mathrm{pl} 3$ in Miller-Dieker syndrome and isolated lissencephaly sequence. Am J Hum Genet Suppl 1991;49:11.

8. Ledbetter SA, Kuwano A, Dobyns WB, Ledbetter DH Microdeletions of chromosome $17 \mathrm{p} 13$ as a cause of isolated lissencephaly. Am J Hum Genet 1992;50:182-189.

9. Pilz DT, Dalton A, Long A, Jaspan T, Maltby EL, Quarrell OWJ. Detecting deletions in the critical region for lissencephaly on 17p13.3 using fluorescent in situ hybridisation and $\mathrm{PCR}$ assay identifying a dinucleotide repeat polymorphism. J Med Genet 1995;32:275-278.

10. Reiner O, Carrozzo R, Shen $Y$, Wehnert M, Faustinella F, Dobyns WB et al. Isolation of a Miller-Dieker lissencephaly gene containing $\mathrm{G}$ protein beta-subunit-like repeats. Nature 1993;364:717-721.

11. Chong SS, Tanigami A, Roschke AV, Ledbetter DH. 14-3-3 epsilon has no homology to LISI and lies telomeric to it on chromosome 17pl3.3 outside the Miller-Dieker chromosome region. Genome Res 1996;6:735-741.

12. Chong SS, Pack SD, Roschke AV, Tanigami A, Carrozzo R, Smith $A C$ et al. A revision of the lissencephaly and MillerDieker syndrome critical regions in chromosome $17 \mathrm{p} 13.3$. Hum Mol Genet 1997;6:147-155.
13. Lo Nigro C, Chong SS, Smith ACM, Dobyns WB, Ledbetter $\mathrm{DH}$. Point mutations and an intragenic deletion in LIS1, the lissencephaly causative gene in isolated lissencephaly sequence and Miller-Dieker syndrome. Hum Mol Genet 1997;6:157-164.

14. Hattori M, Adachi H, Tsujimoto M, Arai N, Inoue K. MillerDieker lissencephaly gene encodes a subunit of brain plateletactivating factor acetylhydrolase. Nature 1994;370:216-218.

15. Fogli A, Lo Nigro C, Renieri A, Fernandes E, Pilz DT, Ledbetter $\mathrm{DH}$, et al. Mutation analysis in the LISI gene of 14 patients with isolated lissencephaly sequence. Am J Hum Genet Suppl 1997;61:A333.

16. des Portes V, Pinard JM, Billuart P, Vinet MC, Koulakoff A, Carrie $A$ et al. A novel CNS gene required for neuronal migration and involved in X-linked subcortical laminar heterotopia and lissencephaly syndrome. Cell 1998;92:51-61.

17. Gleeson JG, Allen KM, Fox JW, Lamperti ED, Berkovic $S$, Scheffer I et al. Doublecortin, a brain-specific gene mutated in human X-linked lissencephaly and double cortex syndrome, encodes a putative signaling protein. Cell 1998;92:63-72.

18. Pinard J-M, Motte J, Chiron C, Brian R, Andermann E, Dulac $O$. Subcortical laminar heterotopia and lissencephaly in two families: a single X linked dominant gene. J Neurol Neurosurg Psychiatry 1994;57:914-920.

19. Dobyns WB, Andermann E, Andermann F, Czapansky-Beilman D, Dubeau F, Dulac O et al. X-linked malformations of neuronal migration. Neurology 1996;47:331-339.

20. des Portes V, Pinard JM, Smadja D, Motte J, Boespflug-Tanguy O, Moutard ML et al. Dominant X linked subcortical laminar heterotopia and lissencephaly syndrome (XSCLH/LIS): evidence for the occurrence of mutation in males and mapping of a potential locus in Xq22. JMed Genet 1997;34:177-183.

21. Ross ME, Allen KM, Srivistava AK, Featherstone T, Gleeson JG, Hirsch B et al. Linkage and physical mapping of X-linked lissencephaly/SBH (XLIS): a gene causing neuronal migration defects in human brain. Hum Mol Genet. 1997;6:555-562.

22. Pilz DT, Matsumoto N, Minnerath S, Mills P, Gleeson JG, Allen $\mathrm{KM}$, et al. $L I S 1$ and $X I I S(D C X)$ mutations cause most classical lissencephaly, but different patterns of malformation. Hum Mol Genet, in press.

23. Ramer JC, Lin AE, Dobyns WB, Ayme S, Pallotta R, Ladda R. Previously apparently undescribed syndrome: shallow orbits, ptosis, coloboma, trigonocephaly, gyral malformations, and mental and growth retardation. Am J Med Genet 1995;57:403-409.

24. Saltzman DH, Krauss CM, Goldman JM, Benacerraf BR. Prenatal diagnosis of lissencephaly. Prenat Diagn 1991;11:139-143.

25. McGahan JP, Grix A, Gerscovich EO. Prenatal diagnosis of lissencephaly: Miller-Dieker syndrome. J Clin Ultrasound 1994;22:560-563. 